\title{
Surface atomic structures, surface energies, and equilibrium crystal shape of molybdenum
}

\author{
J. G. Che* and C. T. Chan \\ Department of Physics, Hong Kong University of Science and Technology, Hong Kong, China \\ W-E. Jian and T. C. Leung \\ Department of Physics, National Chung Cheng University, Taiwan, Republic of China
}

(Received 13 August 1997)

\begin{abstract}
Using first-principles calculations, we calculated the atomic structures and surface energies of molybdenum surfaces in the (100), (110), (111), and (211) orientations. The equilibrium crystal shape of molybdenum is then found using the Wulff construction. We find that all four orientations appear on the Wulff plot and hence they are stable. [S0163-1829(98)06803-9]
\end{abstract}

\section{INTRODUCTION}

The surface properties of the isoelectronic transition metals tungsten and molybdenum have attracted a lot of attention for many years because of their interesting physical and catalytic properties. ${ }^{1-11}$ In addition to being easy to clean, Mo and W surfaces exhibit surface states, surface reconstructions, as well as interesting electronic and structural changes during chemisorption. They have thus been favorite platforms for studying surface phenomena for many decades. They are both body-centered-cubic metals with nearly identical lattice constants (3.15 $\AA$ for Mo and $3.16 \AA$ for W) and have rather similar bulk and surface properties. While the tungsten surface in various orientations has been investigated extensively by both a variety of theoretical methods and surface techniques, comparatively less detailed information about the molybdenum surfaces is available. ${ }^{1-11}$ In many respects, the structural properties of the molybdenum surface are similar to those of tungsten, but there are also subtle differences. For example, the clean (100) tungsten surface shows a $(\sqrt{2} \times \sqrt{2}) R 45^{\circ}$ reconstruction, while more complex reconstructions of the clean $\mathrm{Mo}(100)$ surface have been observed. $^{1,2}$ For a long time, the Mo(100) surface was thought to reconstruct from a $1 \times 1$ structure at room temperature to an incommensurate $c(2.2 \times 2.2)$ structure when cooled below $220 \mathrm{~K}^{1,2}$ The driving mechanism for these reconstructions and the difference between $\mathrm{W}$ and Mo have attracted considerable attention and have been the focus of many experimental and theoretical studies. Recently, Daley et al. found that $\mathrm{Mo}(100)$ forms a commensurate $c(7 \sqrt{2} \times \sqrt{2}) R 45^{\circ}$ at low temperature. ${ }^{12}$

The (110) tungsten and molybdenum surfaces are also interesting for their differences. While the W(110) surface has been observed to reconstruct upon hydrogen adsorption via a uniform lateral shift along the $\left[\begin{array}{lll}0 & \overline{1} & \overline{1}\end{array}\right]$ direction of the surface layer relative to the second layer, ${ }^{13}$ the $\operatorname{Mo}(110)$ surface was not observed to reconstruct. ${ }^{14}$

For Mo, rougher surfaces such as the (111) and (112) have received little attention in theoretical studies, but these orientations are actually important since they may appear in the Wulff plot and thus may be important in governing the equilibrium crystal shape. In the past few years, the stability of the Mo(111) and $\mathrm{W}(111)$ surfaces upon the adsorption of metallic overlayers has been carefully and systematically studied. ${ }^{15,16}$ It was found that some metal overlayers (e.g., $\mathrm{Pt}$ ) can drive the $\mathrm{Mo}(111)$ surface to be unstable and cause the substrate to facet to the $\{112\}$ orientations. A careful study of the "rougher' (111) and (112) surfaces is a necessary first step in understanding these interesting and complex faceting phenomena.

Through a series of first-principles total-energy calculations, we present in this work the results of the atomic structures and surface energies of the Mo surfaces at several orientations and the equilibrium crystal shape of molybdenum at low temperature. We calculated the atomic relaxations of the surface atoms and surface energy for (100), (110), (111), and (211) molybdenum surfaces. The equilibrium shape of a Mo crystallite is then found using the Wulff construction, ${ }^{17}$ assuming that these four orientations are those that appear on the Wulff plot. This is, to our knowledge, the first construction of the equilibrium crystal shape of a bcc metal using first-principles results.

\section{METHOD OF CALCULATION}

The first-principles calculations were done within the local-density-functional formalism ${ }^{18,19}$ together with normconserving pseudopotentials. ${ }^{20}$ The wave functions are expanded by means of a mixed basis set consisting of plane waves with kinetic energy $(\mathbf{k}+\mathbf{G})^{2}$ up to a certain cutoff energy, plus a set of localized numerical functions centered at atomic sites to describe the more tightly bound $d$ orbitals. $^{21}$ This approach has been successfully applied to the electronic and structural properties of many transition metals. $^{22}$ The shape of the numerical orbitals are chosen to optimize the total energy and when that is done, total energies can be converged with a relatively small set of plane waves. In our calculations we used a plane-wave basis with kinetic energy up to $11.5 \mathrm{Ry}$. For the molybdenum bulk, this approach gives a lattice constant $a_{0}=3.16 \AA$ and a bulk modulus $B_{0}=2.8 \mathrm{MBar}$. It agrees well with experimental data $\left(a_{0}^{\text {expt }}=3.15 \AA\right.$ and $\left.B_{0}^{\text {expt }}=2.73 \mathrm{MBar}\right)$.

The surfaces were simulated by the standard slab geometry. Slabs of eleven atomic layers are used, separated by a vacuum of $9.5 \AA$, and repeated periodically along the normal 

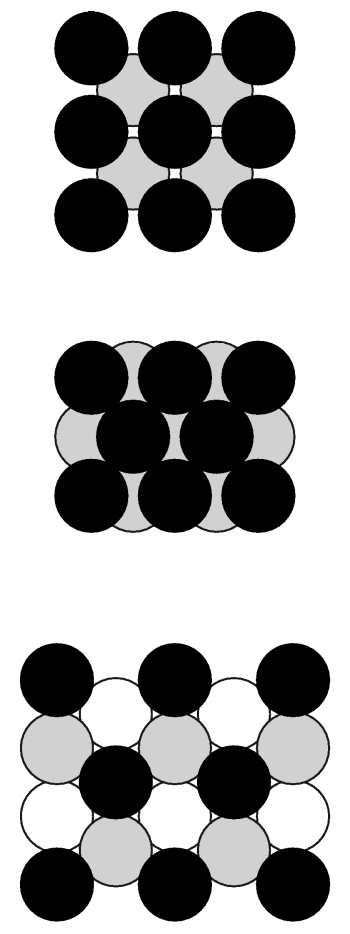

FIG. 1. Top view of the bcc (100), (110), (111), and (211) surfaces. Atoms in the first, second, and third layers are represented by black, gray, and white circles, respectively.

direction of surfaces. For the k-point sampling in the surface calculations, a uniform grid of not less than 100 points in the surface Brillouin zones has been used. We restrict ourselves to $1 \times 1$ surface unit cells, and within that constraint, all the atoms in the unit cell were fully relaxed by computing the Hellmann-Feynman forces on the atoms. Relaxation of atomic positions is facilitated by a force matrix, which is initially taken to be a diagonal matrix with force constants estimated from the Debye temperature. The force matrix is then updated during the course of atomic relaxations using a modified Broyden scheme. ${ }^{23}$ The whole procedure is accelerated by a force-corrector scheme ${ }^{24}$ and the relaxation stops when the maximum of the remaining forces is smaller than $0.03 \mathrm{eV} / \AA$.

\section{RESULTS AND DISCUSSIONS}

\section{A. Atomic structures}

The top views of the (100), (110), (111), and (211) surfaces of a bcc crystal are shown in Fig. 1. The distribution of the nearest neighbors (NN's) and next-nearest neighbors (NNN's) at various layers for a surface atom are listed in Table I. Keeping in mind that an atom in a bcc crystal has
TABLE I. Distribution of the nearest (NN) and next-nearest (NNN) neighbors for a surface atom at different orientations. The top layer is the called first layer. The first two lines mean that for the (100) surface, a surface atom has four NN's at layer 2, four NNN's at layer 1 , and so on.

\begin{tabular}{lccc}
\hline \hline Orientation & Neighbor & Number of atoms & Layer \\
\hline$(100)$ & NN & 4 & 2 \\
& NNN & 4 & 1 \\
& & 1 & 3 \\
\hline$(110)$ & NN & 4 & 1 \\
& & 2 & 2 \\
& NNN & 2 & 1 \\
& & 2 & 2 \\
\hline$(111)$ & NN & 3 & 2 \\
& & 1 & 4 \\
& NNN & 3 & 3 \\
\hline$(211)$ & NN & 2 & 1 \\
& & 2 & 2 \\
& $N$ & 1 & 3 \\
& & 2 & 3 \\
\hline \hline
\end{tabular}

eight NN atoms at a distance of $(\sqrt{3} / 2) a$ and six NNN atoms at a distance of $a$, where $a$ is the lattice constant, the number of NN and NNN broken bonds can be deduced readily from Table I. We note that structural information of bcc systems are also documented elsewhere. ${ }^{25}$

The calculated atomic relaxations of the (100), (110), (111), and (211) Mo surfaces are given in Table II, where we give the percentage change of the interlayer spacings for various orientations. Available theoretical and experimental results are quoted for comparison. In the table $\Delta_{i j}$ denotes the percentage change of the interlayer distance between the $i$ th and $j$ th layers. In the full-potential linear muffin-tin orbital (FP-LMTO) calculations quoted in the table, sevenlayer slabs were used and only the outermost layer is relaxed, while in our calculations all eleven layers of slabs are relaxed. The experimental data are for the reconstructed Mo(100) surface ${ }^{26}$ and unreconstructed $\mathrm{Mo}(110)$ surface. $^{27}$ As expected, surface orientations with a larger number of missing neighbors have larger relaxation of the interlayer spacings. Except for the (211) surface, symmetry requires that the atoms have relaxations only along the surface normal ( $z$ direction) since we have adopted a $1 \times 1$ surface unit cell. However, the atoms on the (211) surface have an additional degree of freedom and can relax along the [111] direction (which is designated as the $y$ direction and the displacement in the $i$ th layer is represented by $\left.\Delta_{y, i}\right)$. The dominant relaxations of the (100), (110), and (211) surfaces are the contraction of the first interlayer spacing, consistent with the generic picture of Smouluchowski smoothing. ${ }^{28,29}$ Relaxation of deeper layers is rather small. The most compact (110) surface has the smallest relative contraction $(4 \%)$ of the interlayer distance between the top two layers, while the contractions are larger for the (100) orientation (11\%) and the (211) orientation $(16 \%)$. This is roughly consistent with the total number of NN and NNN bonds broken: four for (110), five 
TABLE II. Layer relaxation given in the percentage change of the unrelaxed interlayer distance for various orientations. $\Delta_{y, i}$ is the lateral displacement (in angstroms) of the $i$ th layer in the [111] direction relative to the atomic positions of a truncated (211) surface. Available data from other studies are also listed for comparison. (GPT denotes generalized pseudopotential theory.)

\begin{tabular}{|c|c|c|c|c|c|c|}
\hline Orientation & Study & $\Delta_{12}$ & $\Delta_{23}$ & $\Delta_{34}$ & $\Delta_{45}$ & $\Delta_{56}$ \\
\hline \multirow[t]{4}{*}{ (100) } & this work & -11.1 & 2.3 & -1.7 & 0.3 & -0.6 \\
\hline & FP-LMTO $^{\mathrm{a}}$ & -9.0 & & & & \\
\hline & $\mathrm{GPT}^{\mathrm{b}}$ & -10.2 & 1.3 & & & \\
\hline & Expt. $^{\mathrm{c}}$ & $-9.5 \pm 3.0$ & $1.0 \pm 2.0$ & & & \\
\hline \multirow[t]{4}{*}{ (110) } & this work & -4.3 & -0.2 & -0.4 & -0.7 & -0.5 \\
\hline & FP-LMTO $^{a}$ & -3.9 & & & & \\
\hline & $\mathrm{GPT}^{\mathrm{b}}$ & -5.8 & 1.8 & & & \\
\hline & Expt. ${ }^{d}$ & $-1.6 \pm 2.0$ & & & & \\
\hline (111) & this work & -18.7 & -20.3 & 13.7 & -3.0 & 1.6 \\
\hline \multirow[t]{2}{*}{ (211) } & this work & -16.2 & 2.9 & -1.9 & 2.2 & -1.8 \\
\hline & & $\Delta_{y, 1}$ & $\Delta_{y, 2}$ & $\Delta_{y, 3}$ & $\Delta_{y, 4}$ & $\Delta_{y, 5}$ \\
\hline (211) & this work & 0.02 & 0.04 & -0.04 & -0.01 & 0.00 \\
\hline
\end{tabular}

${ }^{\mathrm{a}}$ Reference 10 .

${ }^{\mathrm{b}}$ Reference 38 .

${ }^{\mathrm{c}}$ Reference 26.

${ }^{\mathrm{d}}$ Reference 27.

for (100), and six for (211). The (111) surface exhibits substantial atomic displacements up to the third layer. The (111) surface is the most open among all the orientations considered and atoms in the top three layers are exposed in the top view of a simple ball-and-stick model. With the significant reduction in the interlayer distances between the top three layers and an increase in distance between the third and fourth layers, the relaxation pattern is such that the top three layers come together, "floating" above the rest of the substrate. In the other orientations, it is essentially the top two layers that are exposed to the vacuum and only the top two layers move towards each other. Holzwarth et al. ${ }^{30}$ have considered the multilayer relaxation pattern for $\mathrm{W}(111)$ thin slabs with first-principles calculations up to seven layers thick and with an embedded-atom model (with angular forces) up to 15 layers. The relaxation pattern is very similar to what we have obtained. Such a relaxation pattern may thus be rather generic for bcc(111).

Our results show that the percentage change of the interlayer spacings decreases in the order $\Delta_{12}(111)>\Delta_{12}(211)$ $>\Delta_{12}(100)>\Delta_{12}(110)$. In more general terms, we may tie the amount of relaxation to the "surface roughness," which is defined ${ }^{31}$ as the inverse of the fraction of the area in one plane occupied by atoms of radii equal to one-half the bulk nearest-neighbor distance. For the (111), (211), (100), and (110) surfaces of bcc crystals, the roughnesses so defined are $2.94,2.08,1.70$, and 1.20, respectively. We hence have a simple picture that the "rougher" surfaces have bigger percentage relaxation of interlayer distances.

\section{B. Surface energies}

The orientational dependence of the surface energy is important since it determines the equilibrium shape of crystals ${ }^{17}$ and governs the stability of a surface. ${ }^{32}$ The surface energy per surface atom $\sigma$ can be calculated from the total energy of a supercell of that surface orientation as

$$
\sigma=\left(E_{\text {slab }}-n E_{\text {bulk }}\right) / 2,
$$

where $E_{\text {slab }}$ is the total energy of the supercell containing $n$ Mo atoms and $E_{\text {bulk }}$ is the total energy of bulk Mo. Each slab is bounded by two surfaces and hence a factor of 2 in the above equation. In this article we will use $\sigma$ to denote the surface energy per surface atom and $\gamma$ to denote the surface energy per unit area.

The calculated surface energies per atom and per unit area for the various orientations are tabulated in Table III, where we also include for comparison previous theoretical and experimental work. The surface energy of a solid at a specific orientation is difficult to measure directly and few reliable results are available. The experimental data of the surface energy in Table III were estimated by Tyson and Miller and by Miedema ${ }^{33}$ using the surface energy in the liquid state, which has been determined accurately. Our results show reasonable agreement with the available results from earlier calculations and the experiments.

TABLE III. Surface energies per unit area $\left(\mathrm{J} / \mathrm{m}^{2}\right)$ and per surface atom $(\mathrm{eV} /$ atom $)$ for $1 \times 1$ Mo surfaces at different orientations.

\begin{tabular}{lcccc}
\hline \hline \multicolumn{1}{c}{ Study } & $(100)$ & $(110)$ & $(111)$ & $(211)$ \\
\hline this work $\left(\mathrm{J} / \mathrm{m}^{2}\right)$ & 3.34 & 2.92 & 3.24 & 3.11 \\
this work $(\mathrm{eV} /$ atom $)$ & 2.09 & 1.29 & 3.51 & 2.38 \\
FP-LMTO $\left(\mathrm{J} / \mathrm{m}^{2}\right)^{\mathrm{a}}$ & 3.52 & 3.14 & & \\
Expt. $\left(\mathrm{J} / \mathrm{m}^{2}\right)^{\mathrm{b}}$ & 2.93 & & & \\
\hline \hline
\end{tabular}

${ }^{\mathrm{a}}$ Reference 10 .

${ }^{\mathrm{b}}$ Reference 33 . 
As expected, the calculated surface energies per surface atom for different surfaces decrease with the roughness of the surface: $\sigma(111)>\sigma(211)>\sigma(100)>\sigma(110)$. In forming the (111), (211), (100), and (110) surfaces, four, three, four, and two nearest-neighbor bonds are cut, respectively, while the number of next-nearest-neighbor bonds that are severed are three, three, one and two, respectively. For bcc crystals, the difference between the nearest-neighbor distance $(\sqrt{3} / 2) a$ and the next-nearest-neighbor distance $a$ is not large. The trend of the surface energies for the various orientation is indeed consistent with the total number of NN and NNN bonds that are broken when the surface is created.

Going from the surface energy per atom to the surface energy per unit area, the variation is much smaller from one orientation to another. Among the orientations considered in this work, the (100) surface has the highest surface energy per unit area and the (110) surface the lowest. From our calculations, the ratio of the surface energies per unit area $\gamma_{h k l} / \gamma_{100}$ are 1.0, 0.97, 0.93, and 0.87 for the (100), (111), (211), and (110) surfaces, respectively, compared with the values $1.0,0.87,0.80$, and 0.71 obtained from a bond-cutting model. ${ }^{34}$ We see that even the simplest bond-cutting models predict correctly the trend of the surface energies. When we compare the numbers, we see that the bond-cutting model overestimates the surface energies of less compact orientations relative to the most compact (110) orientation. Bondcutting or lattice models do not allow for the energy gained by relaxations. Since less compact surfaces usually have more relaxations, it is not surprising that the empirical models will overestimate the surface energies of less compact orientations.

In the past few years it was observed that ultrathin metal films adsorbed on Mo(111) and W(111) can induce the substrate to facet, exposing facets of $\{211\}$ orientations, but the clean (111) surface is stable (see, e.g., Ref. 15). According to thermodynamic theories, ${ }^{32}$ faceting from (111) to (112) is thermodynamically favorable when

$$
\gamma_{112} / \cos (\theta)-\gamma_{111}<0,
$$

where $\theta=19.47^{\circ}$ is the angle between the [111] and the [112] orientations and $\gamma$ is the surface formation energy per unit area. The $\cos (\theta)$ factor takes care of the increase in area when faceting occurs.

Our results indicate that the (211) surface has a lower surface energy per unit area than (111), but Eq. (2) is not satisfied. The anisotropy is not large enough to compensate for the $6 \%$ increase in total area due to faceting. The clean (111) surface should thus be thermodynamically stable against faceting, consistent with experimental results. The surface energy and its anisotropy can change significantly upon chemisorption, and overlayer covered surfaces can behave very differently from the clean surface. ${ }^{35}$

\section{Equilibrium crystal shape}

The equilibrium crystal shape (ECS) can be obtained by minimizing the total surface free energy for a fixed crystal volume. ${ }^{17}$ The equilibrium crystal shape is determined if the orientational dependence of the surface free energy (surface energy at $T=0$ ) is known. According to the procedure of the Wulff construction, a polar plot of surface energy is made. A

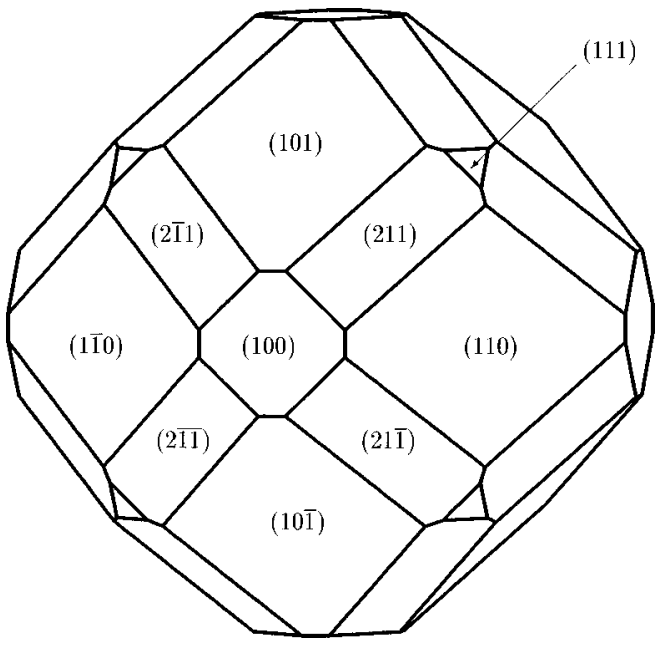

FIG. 2. Equilibrium crystal shape of Mo constructed with surface energies calculated from first principles.

vector whose length is equal to the surface energy $\gamma$ in this orientation is chosen and then a plane perpendicular to the vector is drawn, passing through the tip of the vector. The inner envelope of all these planes gives the equilibrium shape of a crystallite under ideal conditions. Crystal planes that are part of this construction are thermodynamically stable.

Under the assumption that only the $\{100\},\{110\},\{111\}$, and $\{211\}$ facets appear on the Wulff plot and using the surface energies we have calculated for these Mo surfaces (Table III), the equilibrium shape for Mo is shown in Fig. 2. We see that facets corresponding to the $\{110\}$ orientations have the largest area, while the $\{111\}$ orientations occupy the smallest area. It was found experimentally that the Mo(111) surface is stable ${ }^{15,16}$ consistent with the fact the $\{111\}$ facets appear on the Wulff plot. However, adsorption of an overlayer of some metals such as $\mathrm{Pd}$ and $\mathrm{Pt}$ on $\mathrm{Mo}(111)$ can induce a faceting transformation, ${ }^{15,16}$ forming triangular pyramids exposing three equivalent $\{112\}$ facets. The same phenomena have been observed in $\mathrm{W}(111) .{ }^{15}$ We see from Fig. 2 that the area occupied by $\{111\}$ is very small. If the surface energy anisotropy is enhanced in favor of the $\{112\}$ orientations upon adsorption, the area corresponding to the $\{112\}$ faces on the ECS will increase, while the corresponding area for the $\{111\}$ faces will shrink and may eventually disappear. The [111] orientated surface would then become unstable and may facet if the kinetic barriers can be overcome. It is almost visually obvious from Fig. 2 that triangular pyramids of $\{112\}$ will form when $\{111\}$ becomes unstable with respect to a faceting transformation.

Although the first-principles calculations and a simple bond-cutting model give the same ordering of the surface energy per unit area for the four orientations we have considered, as we have noted in Sec. III B, the equilibrium crystal shape from these two approaches can be rather different. We show in Fig. 3 the equilibrium crystal shape of a bcc solid with surface energy anisotropy corresponding to a simple bond-cutting model (with the ratios of surface energies given in the previous section). Only $\{110\}$ and $\{112\}$ facets appear on the ECS and the shape is very different from the first-principles results. This will have certain physical implications. For example, such an equilibrium crystal shape 


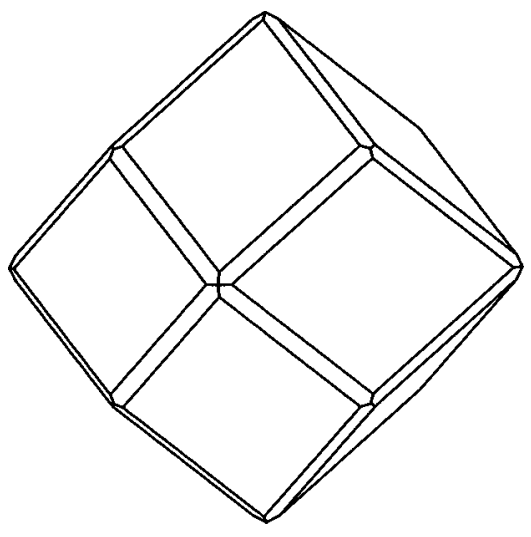

FIG. 3. Equilibrium crystal shape of Mo constructed from a bond-cutting model (see the text).

would mean that a clean $\operatorname{Mo}(111)$ surface is unstable with respect to a faceting transformation to the $\{112\}$ orientations, which is apparently not the case experimentally. This is a consequence of the overestimation of the surface energies of the less compact surfaces in bond-cutting models.

We remark that, in principle, the Wulff plot should be determined by the surface energy at all possible orientations, while we have first-principles information for four principal orientations only. This restriction is probably not a serious problem since for most of the generic models, ${ }^{36}$ it is these four orientations that appear on the equilibrium crystal shape of a bcc crystal at $T=0$. It is possible to take one step further to fit the available information to an empirical model and then extrapolate from our calculated results to other orientations. This approach has been adopted by Wei and Chou, ${ }^{37}$ who have considered the equilibrium crystal shape for several fcc metals with a model based on surface energies from various $a b$ initio calculations. This procedure seems to work very well for fcc metals. The relaxations for the bcc surfaces are comparatively larger, and higher index surfaces will have even larger relaxations. Since the model extrapolations ignore relaxation effects, it is not clear at this point whether such a process will give us additional reliable information in addition to the first-principles results for bcc surfaces.

Another subtlety is that we have not considered reconstructions and all our results pertain to a fully relaxed $p(1 \times 1)$ surface. It is well known that the (100) surfaces of Mo and $\mathrm{W}$ reconstruct and we cannot rule out the possibility that other orientations such as (111) and (112) also show some reconstructions. For the (112) surface, we have taken into account the lateral shifting of the layers, which is not forbidden by symmetry; but possible relative displacement of atoms within one surface layer has not been considered. However, calculations have shown that for the Mo(100) surface, the energy gained by surface atomic rearrangements mainly comes from the relaxation normal to the surface and the energy gained by reconstruction on top of the relaxation is small. ${ }^{3}$ For example, we compared the total energy of a fully reconstructed Debe-King model within a $c(2 \times 2)$ surface unit cell (both vertical and lateral degrees of freedom are fully relaxed) and that of a fully relaxed $p(1 \times 1)$ $\mathrm{Mo}(100)$ surface (vertical relaxation only) and we found that the surface energy of the $c(2 \times 2)$ is more favorable than the fully relaxed $p(1 \times 1)$ by about $1 \mathrm{mRy}$ per surface atom. ${ }^{39}$ Such a small gain in energy has little effect on our results. The ordering of surface energies will be the same and taking that into account will only increase the area of the $\{100\}$ surfaces slightly on the Wulff plot.

\section{CONCLUSIONS}

In conclusion, we have presented a theoretical study of the equilibrium atomic structures of the Mo (100), (110), (111), and (211) surfaces, using first-principles total-energy and force calculations. Our results found that rougher surfaces tend to have larger relaxations and higher surface energies per surface atom. Atomic relaxations for different orientations are mainly dominated by the reduction of the separation between the top two layers, except for the (111) surfaces where the top three layers come together. This can be rationalized because the (111) surface has three layers of atoms exposed, while other orientations have two. We obtained the equilibrium crystal shape of Mo (at $T=0$ ), which is probably the first time that the equilibrium crystal shape of a bcc crystal has been determined entirely from firstprinciples results without mapping to fixed-lattice models. All four orientations appear on the Wulff plot and we found that the Mo(111) surface is thermodynamically stable against forming facets in the $\{211\}$ orientations according to the Herring criteria.

\section{ACKNOWLEDGMENTS}

We thank Professor T. Madey for discussions about the issue of faceting. This work was supported by RGC Hong Kong through Grant No. HKUST694/96P, and we thank AMRI-HKUST for computer time. T.C.L. and W.E.J. were supported by National Science Council Grant No. 86-2112M-194-011 and a grant of computer time at the National Center for High-Performance Computing.
*Permanent address: Fudan T. D. Lee Physics Laboratory and Department of Physics, Fudan University, Shanghai, China.

${ }^{1}$ M. K. Debe and D. A. King, Phys. Rev. Lett. 30, 708 (1977).

${ }^{2}$ T. E. Felter, R. A. Barker, and P. J. Estrup, Phys. Rev. Lett. 38, 1138 (1977).

${ }^{3}$ X. W. Wang, C. T. Chan, K. M. Ho, and W. Weber, Phys. Rev. Lett. 60, 2066 (1988).

${ }^{4}$ K. Jeong, R. H. Gaylord, and S. D. Kevan, Phys. Rev. B 39, 2973 (1989).

${ }^{5}$ E. Hulpke and D. M. Smilgies, Phys. Rev. B 40, 1338 (1989).

${ }^{6}$ E. Hulpke and D. M. Smilgies, Phys. Rev. B 43, 1260 (1989).
${ }^{7}$ K. E. Smith and S. D. Kevan, Phys. Rev. B 43, 1831 (1991).

${ }^{8}$ K. E. Smith and S. D. Kevan, Phys. Rev. B 43, 3986 (1991).

${ }^{9}$ K. E. Smith, G. S. Elliott, and S. D. Kevan, Appl. Surf. Sci. 48/49, 135 (1991).

${ }^{10}$ M. Methfessel, D. Hennig, and M. Scheffler, Phys. Rev. B 46, 4816 (1992).

${ }^{11}$ E. Hulpke and J. Lüdecke, Surf. Sci. 287/288, 837 (1993).

${ }^{12}$ R. S. Daley, T. E. Felter, M. L. Hildner, and P. J. Estrup, Phys. Rev. Lett. 70, 1295 (1993).

${ }^{13}$ J. W. Chung, S. C. Ying, and P. J. Estrup, Phys. Rev. Lett. 56, 749 (1986). 
${ }^{14}$ M. Altmann, J. W. Chung, P. J. Estrup, J. M. Kosterlitz, J. Prybyla, D. Sahu, and S. C. Ying, J. Vac. Sci. Technol. A 5, 1045 (1987).

${ }^{15}$ T. Madey, J. Guan, C. H. Nien, C. Z. Dong, H. S. Tao, and R. A. Campbell, Surf. Sci. Rev. Lett. 3, 1315 (1996).

${ }^{16}$ J. Guan, Ph.D. thesis, Rutgers University, 1994.

${ }^{17}$ G. Wulff, Z. Krist. Mineral 34, 449 (1901).

${ }^{18}$ P. Hohenberg and W. Kohn, Phys. Rev. 136, B864 (1964); W. Kohn and J. S. Sham, ibid. 140, A1133 (1965).

${ }^{19}$ L. Hedin and B. I. Lundqvist, J. Phys. C 4, 2064 (1971).

${ }^{20}$ D. R. Hamann, M. Schlüter, and C. Chiang, Phys. Rev. Lett. 43, 1494 (1979); G. B. Bachelet and M. Schlüter, Phys. Rev. B 25, 2103 (1982).

${ }^{21}$ S. G. Louie, K. M. Ho, and M. L. Cohen, Phys. Rev. B 19, 1774 (1979); C. Elsasser, N. Takeuchi, K. M. Ho, C. T. Chan, P. Braun, and M. Fahnle, J. Phys.: Condens. Matter 2, 4371 (1980).

${ }^{22}$ See, e.g., C. T. Chan, K. M. Ho, and K. P. Bohnen, in Handbook of Surface Science, edited by W. N. Unertl (Elsevier, Amsterdam, 1996), Vol. I, Chap. 3.

${ }^{23}$ D. D. Johnson, Phys. Rev. B 38, 12807 (1988).

${ }^{24}$ C. T. Chan, K. P. Bohnen, and K. M. Ho, Phys. Rev. B 47, 4771 (1993).
${ }^{25}$ See, e.g., K. L. Murphy and C. Rottman, Phys. Rev. B 42, 680 (1990).

${ }^{26}$ L. J. Clarke, Surf. Sci. 91, 131 (1980).

${ }^{27}$ L. Morales de la Garza and L. J. Clarke, J. Phys. C 14, 5391 (1981).

${ }^{28}$ R. Smouluchowski, Phys. Rev. 60, 661 (1941).

${ }^{29}$ M. W. Finnis and V. Heine, J. Phys. F 4, L37 (1974).

${ }^{30}$ N. A. W. Holzwarth, J. A. Chervenak, C. J. Kimmer, Y. Zheng, W. Xu, and J. Adams, Phys. Rev. B 48, 12136 (1993).

${ }^{31}$ J. Sokolov, F. Jona, and P. M. Marcus, Solid State Commun. 49, 307 (1984).

${ }^{32}$ C. Herring, Phys. Rev. 82, 87 (1951).

${ }^{33}$ W. R. Tyson and W. A. Miller, Surf. Sci. 62, 267 (1977); A. R. Miedema, Z. Metallkd. 69, 287 (1978).

${ }^{34}$ J. K. Mackenzie and J. F. Nicholas, J. Phys. Chem. Solids 23, 197 (1962).

${ }^{35}$ J. G. Che, C. T. Chan, J. Kuo, and T. C. Leung, Phys. Rev. Lett. 79, 4230 (1997).

${ }^{36}$ See, e.g., A. C. Shi, Phys. Rev. B 36, 9068 (1987).

${ }^{37}$ S. Q. Wei and M. Y. Chou, Phys. Rev. B 50, 4859 (1994).

${ }^{38}$ J. A. Moriarty and R. Phillips, Phys. Rev. Lett. 66, 3036 (1991).

${ }^{39}$ For the $c(2 \times 2)$ calculations, we used seven-layer slabs and $36 k$ points in the irreducible Brillouin zone. 